\title{
Nicotine and methyl vinyl ketone, major components of cigarette smoke extracts, increase protective amyloid- $\beta$ peptides in cells harboring amyloid- $\beta$ precursor protein
}

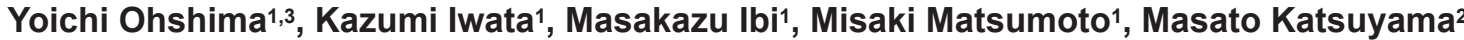 \\ and Chihiro Yabe-Nishimura1 \\ ${ }^{I}$ Department of Pharmacology, Kyoto Prefectural University of Medicine, 465 Kajii-cho, Kawaramachi-Hirokoji, \\ Kamikyo-ku, Kyoto 602-8566, Japan \\ ${ }^{2}$ Radioisotope Center, Kyoto Prefectural University of Medicine, 465 Kajii-cho, Kawaramachi-Hirokoji, \\ Kamikyo-ku, Kyoto 602-8566, Japan \\ ${ }^{3}$ Department of Neurology, Kyoto Yamashiro General Medical Center, 1-27 Kizu station, Kizugawa, Kyoto 619-0214, Japan
}

(Received November 15, 2017; Accepted February 1, 2018)

\begin{abstract}
The increased ratio of longer amyloid- $\beta$ (A $\beta 1-42)$ /shorter amyloid- $\beta$ (A $\beta 1-40)$ peptides, generated from amyloid precursor protein (APP), is known to promote the development of Alzheimer's disease (AD). To investigate the role of smoking in $\mathrm{A} \beta$ production, we determined the production of $\mathrm{A} \beta$ species in the presence of nicotine or methyl vinyl ketone (MVK), major components of cigarette smoke extracts, in Flp-In ${ }^{\mathrm{TM}}$ T-REx ${ }^{\mathrm{TM}}-293$ (T-REx293) cells harboring a single copy of human APP. While treatment with nicotine or MVK did not affect the amount of APP, the levels of A $\beta 1-40$ in the culture media were significantly increased. On the other hand, the levels of $A \beta 1-42$ were unaltered by nicotine or MVK treatment. The A $\beta 1-42 / A \beta 1-40$ ratio was therefore attenuated by cigarette smoke extracts. Similar results were obtained in T-REx293 cells harboring APP of Swedish- or London-type mutation linked to familial AD. T-REx293 cells expressed the nicotinic acetylcholine receptor (nAchR) and tubocurarine, an nAChR antagonist, completely blocked the effects of nicotine. Treatment with nicotine significantly elevated cellular levels of $\beta$-secretase that cleaves APP prior to A $\beta$ generation. Taken together, a protective role of nicotine against $\mathrm{AD}$ pathology was suggested by enhanced extracellular $\mathrm{A} \beta 1-40$ production, which may suppress $\mathrm{A} \beta$ fibrillogenesis.
\end{abstract}

Key words: Alzheimer's disease, Amyloid- $\beta$, Amyloid precursor protein, Methyl vinyl ketone, Nicotine

\section{INTRODUCTION}

Alzheimer's disease (AD) is the most prevalent neurodegenerative disorder. It is characterized pathologically by the emergence of senile plaques and neurofibrillary tangles in the brain. The senile plaques are extracellular and intracellular protein aggregates composed of amyloid $\beta(A \beta)$. Genetic studies linked early-onset familial $\mathrm{AD}$ (FAD) to various mutations in the genes encoding $\beta$-amyloid precursor protein (APP) as well as presenilin 1 and 2 (PS1 and PS2), the catalytic subunits of the $\gamma$-secretase complex, which liberates A $\beta$ peptides. Most of these mutations share a common phenotype by showing an absolute or relative increase in the production of the highly fibrillogenic A $\beta 1-42$ peptide (Scheuner et al., 1996; Selkoe, 1997).
Experimental studies on AD have been largely conducted using cellular and animal models overexpressing the mutant genes of APP. However, these models represent artificial phenotypes because they overproduce not only A $\beta$ peptides, but also APP and its fragments (Nilsson et al., 2014; Saido, 2013; Saito et al., 2014). Concerns have thus been raised regarding these overexpression models. In order to overcome such a problem, we established a regulated cellular system stably expressing a single copy of wild-type or mutant $A P P$ under relatively physiological conditions using FLp-In ${ }^{\mathrm{TM}} \mathrm{T}-\mathrm{REx}^{\mathrm{TM}}{ }_{-}$ 293 system.

While the effects of smoking on the development of $\mathrm{AD}$ were variable in different epidemiological studies, protective effects of nicotine have been documented in $\mathrm{A} \beta$-induced neuronal cell death as well as in the accumu-

Correspondence: Chihiro Yabe-Nishimura (E-mail: nchihiro@koto.kpu-m.ac.jp) 
lation of $\mathrm{A} \beta$ (Kihara et al., 1998; Ono et al., 2002; Shaw et al., 2002). Such effects of nicotine were mediated by nicotinic acetylcholine receptor (nAchR), a pentameric ligand-gated cation channel, composed of alpha and beta subunits. The $\alpha 7$ and $\alpha 4 \beta 2$ subtypes, the majority of the nicotinic receptor subtypes expressed in the brain, have been demonstrated to be involved in cognitive function and interact with $\mathrm{A} \beta$ (Lombardo and Maskos, 2015).

In the present study, we examined the effects of major components of cigarette smoke extract on the production of extracellular and intracellular $\mathrm{A} \beta$ species. We herein report that nicotine increased generation of extracellular A $\beta 1-40$ originated from not only wild-type APP but also mutant APP of FAD by increasing the level of $\beta$-secretase that cleaves APP prior to $A \beta$ generation. Increased $A \beta 1-40$ may reduce the ratio of $A \beta 1-42 / A \beta 1-40$ peptides, thereby protecting against $A \beta 1-42$-induced fibrillogenesis.

\section{MATERIALS AND METHODS}

\section{Reagents}

Nicotine, 3-buten-2-one (methyl vinyl ketone; MVK), and a rabbit polyclonal antibody to the APP C-terminal (A8717) were purchased from Sigma-Aldrich (St. Louis, MO, USA). A rabbit polyclonal antibody to BACE1 was from Abcam (Cambridge, UK). A rabbit polyclonal antibody to phospho APP (Thr668, \#3823) and a $\beta$-actin antibody (\#4970) were from Cell Signaling Technology (Danvers, MA, USA). A goat anti-mouse IgG-horseradish peroxidase (HRP) antibody (sc-2005) and a goat anti-rabbit IgG-HRP antibody (sc-2054) were from Santa Cruz Biotechnology (Dallas, TX, USA). Hygromycin B (400052) was from Calbiochem (La Jolla, CA, USA). SuperSignal West Femto Maximum Sensitivity Substrate (\#34095) and SuperSignal ELISA Femto Maximum Sensitivity Substrate (\#37075) were obtained from Thermo Fisher Scientific (Waltham, MA, USA). Ham's F-12 medium (17458-65) was from Nacalai Tesque (Kyoto, Japan) and penicillin-streptomycin (15140-122) from Gibco (Palo Alto, CA, USA). Fetal bovine serum (SH30910.03) was obtained from Hyclone (Chicago, IL, USA).

\section{Vector construction}

To introduce human wild-type APP695 cDNA and Swedish-mutant APP695 cDNA (K670NM671L) into a pcDNA5/FRT/TO vector (Invitrogen, Carlsbad, CA, USA), pEF-BOS vectors harboring wild- or Swedish-type $A P P$ (gifted from Dementia and Higher Brain Function Research, Tokyo Metropolitan Institute of Medical Science) were used as templates to amplify $A P P$ cDNA by
PCR. Using the Rapid DNA Ligation Kit (Roche, Basel, Switzerland), PCR products were inserted into the multicloning sites of pcDNA5/FRT/TO. London (V717I) mutation was introduced into the wild-type $A P P$ plasmid by site-directed mutagenesis using KOD-Plus-Mutagenesis Kit (Toyobo, Osaka, Japan). Following confirmation by sequence analyses, vectors harboring human wild-, Swedish-, or London-type $A P P$ were supplied for transfection.

\section{Cell culture and transfection}

Flp- $\operatorname{In}^{\text {TM }}$ T-REx ${ }^{\text {TM }}-293$ cells (T-REx 293, Invitrogen) were cultured in Ham's F-12 medium containing 10\% fetal bovine serum. Cells were co-transfected with a pOG44 vector (Invitrogen) and pcDNA5/FRT/TO vector coding for wild-type or mutant $A P P$ with lipofectamine LTX as described (Watanabe-Hosomi et al., 2012). Cells transfected with the empty pcDNA5/FRT/TO were used as a control. Stable cell lines were selected in the presence of $100 \mu \mathrm{g} / \mathrm{mL}$ hygromycin B. To express exogenous $A P P, \mathrm{~T}-\mathrm{REx} 293$ cell lines were incubated with $1 \mu \mathrm{g} / \mathrm{mL}$ tetracycline. In this system, homogeneous levels of $A P P$ expression can be expected by targeted integration of the expression vector to the same locus in every cell.

\section{Sample preparation for immunoblotting and ELISA}

Cells were cultured in serum-free Ham's F-12 medium containing tetracycline in 6 -well dishes at $37^{\circ} \mathrm{C}$ for 3 days with or without nicotine or MVK. Culture media were collected with a protease inhibitor cocktail (0408011, Nacalai Tesque) and cells were dissolved in RIPA buffer (50 mM Tris- $\mathrm{HCl}$ buffer $\mathrm{pH} 7.6,150 \mathrm{mM} \mathrm{NaCl}$, $1 \%$ Nonidet P40, $0.5 \%$ sodium deoxycholate) containing a protease inhibitor (08714-04, Nacalai Tesque). Following centrifugation, the protein amount in the supernatant fraction was determined by a BCA protein assay kit (Thermo Fisher Scientific).

\section{Immunoblotting}

Cell lysates with $20 \mu \mathrm{L}$ lithium dodecyl sulfate (LDS) sample loading buffer (Invitrogen) were loaded on $12 \%$ Bis-Tris Protein Gels (Invitrogen) and transferred to PVDF membranes (0.45 $\mu \mathrm{m}$, Merck, Darmstadt, Germany). These membranes were pretreated with Blocking One (Nacalai Tesque) at room temperature and incubated with an anti-APP-C, anti- $\beta$-secretase (BACE1), anti-phosphorylated APP (Thr668), or anti- $\beta$-actin antibody. Following incubation with a goat anti-mouse or rabbit IgG -HRP antibody at room temperature, the membranes were treated with chemiluminescent reagents, and proteins were detected using ImageQuant LAS 4000 mini (GE Healthcare, Buckinghamshire, UK). The pixel density of each band 
Cigarette smoke extracts increase protective amyloid- $\beta$ peptides

was quantified by ImageJ software (NIH). As for the amount of total APP, the summed density of two bands between 110 160 kDa was calculated.

\section{ELISA for monomeric $A \beta$ species and $A \beta$ oligomers}

The levels of $A \beta 1-40$ and $A \beta 1-42$ in the culture media were measured using Human $\beta$ Amyloid (1-40) ELISA kit (292-62301) and $\beta$ Amyloid (1-42) ELISA kit (298-62401) (Wako, Osaka, Japan), respectively, in accordance with the manufacturer's instructions.

The levels of $A \beta$ oligomers (A $\beta O s$ ) were measured using the A $\beta$ Os-specific ELISA system (Fukumoto et al., 2010; Kasai et al., 2012). In brief, 96-well plates were incubated with carbonate buffer containing the anti-A $\beta$ monoclonal antibody BAN50 $(10 \mu \mathrm{g} / \mathrm{mL})$ at $4{ }^{\circ} \mathrm{C}$ overnight. Culture media, cell lysates, or standard reagents containing $\mathrm{A} \beta \mathrm{O}$ s were applied to each well, and incubated at $4^{\circ} \mathrm{C}$ overnight. The detector antibody, the HRPconjugated $\mathrm{Fab}^{\prime}$ fragment of BAN50 diluted 1:2500 with buffer $\mathrm{C}$ (heat-inactivated $20 \mathrm{mM}$ phosphate buffer, $\mathrm{pH} 7.0$, containing $0.2 \%$ protease-free BSA, 2 mM EDTA, $400 \mathrm{mM} \mathrm{NaCl}$, and $0.05 \%$ sodium merthiolate) was added to the wells, and incubated at room temperature for $3 \mathrm{hr}$. Finally, the chemiluminescent substrates (SuperSignal ELISA Femto Maximum Sensitivity Substrate; Thermo Fisher Scientific) were added to the wells.

The enzymatic products were measured by a microplate spectrophotometer (SpectraMax Plus384; Molecular Devices, Osaka, Japan) at $450 \mathrm{~nm}\left(\mathrm{OD}_{450}\right)$ for TMB substrate or with a luminometer (SpectraMaxL; Molecular Devices, Osaka, Japan) for the chemiluminescent substrate. The levels of $A \beta 1-40, A \beta 1-42$, and $A \beta O$ s were corrected by the level of total APP quantified as described above.

\section{Detection of $n A c h R$ subunits}

To examine the expression of $n A c h R$ in $T-R E x$ 293 cells, PCR was conducted with KOD-Plus-Neo (TOYOBO) according to the protocol, and the products were analyzed by electrophoresis. Primers used to detect nAchR $\alpha 7$ subunit were: 5'-GAC TGT TCG TTT CCC AGA TGG-3' and 5' - ACG AAG TTG GGA GCC GAC ATC A-3'. Primers used to detect nAchR $\alpha 4$ subunit were: 5'-CTC TCG CAA CAC CCA CTC GG-3' and 5'-AGC AGG CTC CCG GTC CCT TCT AG-3'. Oligonucleotide primers were acquired from Life Technologies (Grand Island, NY, USA).

\section{Statistical analysis}

Results were expressed as means +/- standard error of the mean. All data were analyzed using a one-way ANOVA followed by Dunnett's test with GraphPad Prism6 (GraphPad Software, La Jolla, CA, USA).

\section{RESULTS}

\section{Nicotine did not affect the expression of APP}

First, the amount of total APP expressed in T-REx 293 cells was verified. Immunoblot analyses indicated that similar levels of APP were expressed in cells harboring wild-type or mutated APP (Fig. 1A). Following the treat-

(A)

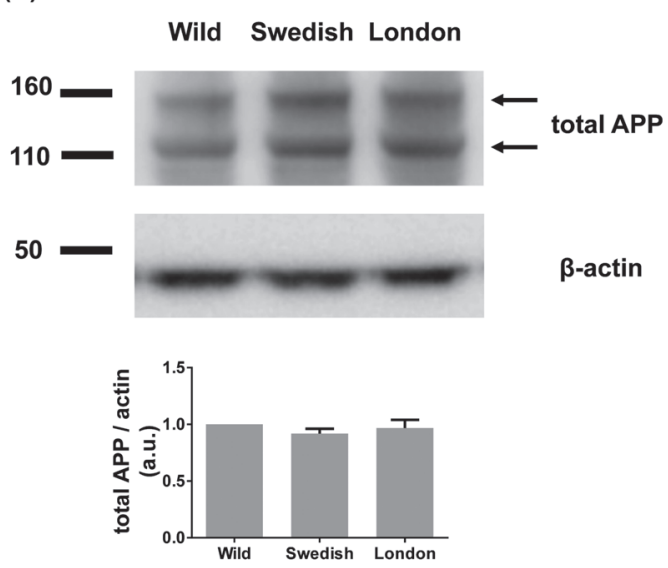

(B)
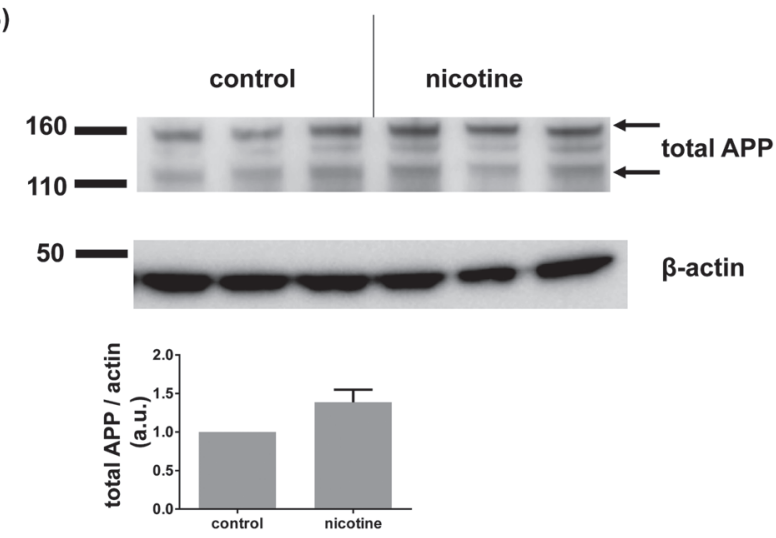

Fig. 1. Nicotine did not affect the expression of APP. (A) The level of total APP in cells harboring wild-type or mutated APP was determined by immunoblotting ( $5 \mu \mathrm{g}$ protein/lane). The summed density of the two bands (arrows) was quantified as the amount of total APP. (B) The level of total APP in cells incubated with or without nicotine $(20 \mu \mathrm{M})$ was determined by immunoblotting. The summed density of the two bands (arrows) was quantified as the amount of total APP. a.u.: arbitrary unit. 
ment with $20 \mu \mathrm{M}$ nicotine for 3 days, the level of APP in cells harboring wild-type $A P P$ tended to increase. However, no significant difference was observed (Fig. 1B).

\section{Nicotine and MVK increased the level of extracellular $A \beta 1-40$}

When the levels of $A \beta$ monomers in the culture media were determined, a dose-dependent increase in A $\beta 1-40$ was demonstrated in cells harboring wild-type APP treated with nicotine. On the other hand, the level of $A \beta 1$ 42 was unaltered (Fig. 2A). Similarly, the level of A $\beta 1$ 40 was significantly elevated in the culture media of cells treated with 100-250 nM MVK, while no significant effect of MVK was observed on the level of $A \beta 1$ 42 (Fig. 2B). A time course study demonstrated that the effect of nicotine became apparent at 3 days after nicotine treatment (Fig. 2C), while the level of $A \beta 1-40$ significantly increased at 5 days after MVK treatment (Fig. 2D).

Consequently, the ratio of $A \beta 1-42$ to $A \beta 1-40$ in the medium was significantly attenuated, or tended to decline, in cells treated with nicotine or MVK (Fig. 3A). In cell lysates, on the other hand, the levels of $A \beta$ monomers were very low and unaltered by nicotine or MVK treatment (Fig. 3B).

The level of extracellular $A \beta 1-40$ was also increased by nicotine or MVK treatment in cells harboring mutant Swedish-type or London-type APP, while the level of A $\beta 1-42$ was unaffected (Fig. 4). Control levels of $A \beta$ monomers derived from mutant $A P P$-transfected cells were much higher than those from cells harboring wildtype $A P P$.

\section{Nicotine-induced increase in $A \beta 1-40$ was mediated by $\mathrm{nAChR}$}

When the expression of nicotinic acetylcholine receptor (nAchR) in T-REx 293 cells was assessed, the $\alpha 7$ as well as $\alpha 4$ subunits of $n A c h R$ were expressed, comparable to SH-SY5Y neuroblastoma-derived cells (Fig. 5A).

As shown in Fig. 5B, an increased level of A $\beta 1-40$ in cells harboring wild-type- $A P P$ was almost completely blocked by tubocurarine, an nAChR antagonist. These findings suggest that enhanced production of $A \beta 1-40$ induced by nicotine was mediated by $\mathrm{nAChR}$.

\section{Nicotine did not affect the levels of extracellular A $\beta$ Os}

Given that $\mathrm{A} \beta \mathrm{O}$ s contribute to neurotoxicity in $\mathrm{AD}$, the amount of $A \beta O$ s in the culture medium was determined by $\mathrm{A} \beta \mathrm{O}$-specific ELISA system. As shown in Fig. 6, there was no difference in the levels of $A \beta O$ s reacted with the anti-A $\beta$ monoclonal antibody in the culture media of cells harboring wild-, Swedish-, or London-type $A P P$ treated with nicotine.

\section{Nicotine increased BACE1 that cleaves APP}

To elucidate the mechanism underlying increased levels of A $\beta 1-40$ in the medium, the level of $\beta$-secretase, BACE1 that cleaves APP within the ectodomain to liberate soluble APP fragment, was examined. As shown in Fig. 7A, the level of BACE1 was significantly increased in cells harboring wild-type $A P P$ treated with nicotine for 3 days.

The phosphorylation of APP at Thr668 is known to facilitate the amyloidogenic processing of APP by both $\beta$ and $\gamma$-secretases (Muresan and Ladescu Muresan, 2015). When the level of phosphorylated APP was examined, no significant alteration was observed in cells treated with nicotine (Fig. 7B).

\section{DISCUSSION}

We herein demonstrated that major components of cigarette smoke extracts, nicotine and MVK, significantly increased the extracellular level of $\mathrm{A} \beta 1-40$ in cells harboring not only wild-type $A P P$ but also mutant $A P P$. On the other hand, extracellular levels of $A \beta O$ s were not affected by the treatment of these components. Given that intracellular levels of $A \beta$ were equivalent to the levels of $A \beta$ in control cells, cigarette smoke extracts apparently promote the production of $A \beta 1-40$ monomer from $A \beta$ to reduce the ratio of extracellular $A \beta 1-42 / A \beta 1-40$. Since a relative increase in $A \beta 1-42$ peptide accelerates the formation of $\mathrm{A} \beta$ amyloid fibrils, a pathogenic component for AD (Hardy and Higgins, 1992; Selkoe, 1991), the decreased ratio of $A \beta 1-42 / A \beta 1-40$ induced by cigarette smoke extracts may play a protective role in the development of AD.

Previous studies were conducted using cellular and animal models that overexpress $A P P$ in order to quantify the amount of $\mathrm{A} \beta$ species relevant to the development of $\mathrm{AD}$. However, the phenotypes demonstrated in mice overexpressing human $A P P$ were found to be artifacts resulting from high levels of APP and its non-A $\beta$ fragments (Saido, 2013). Concerns have thus been raised regarding these overexpression models. In order to avoid these issues, we used a cellular system expressing a single copy of $A P P$, and compared the levels of $\mathrm{A} \beta$ monomers and $A \beta O$ s in wild-type and mutant $A P P$-transfected cells under relatively physiological conditions.

In this study, no change in extracellular $A \beta O$ s levels was demonstrated in cells treated with nicotine despite the reduced ratio of extracellular $A \beta 1-42 / A \beta 1-40$. The ELI- 
Cigarette smoke extracts increase protective amyloid- $\beta$ peptides

(A)
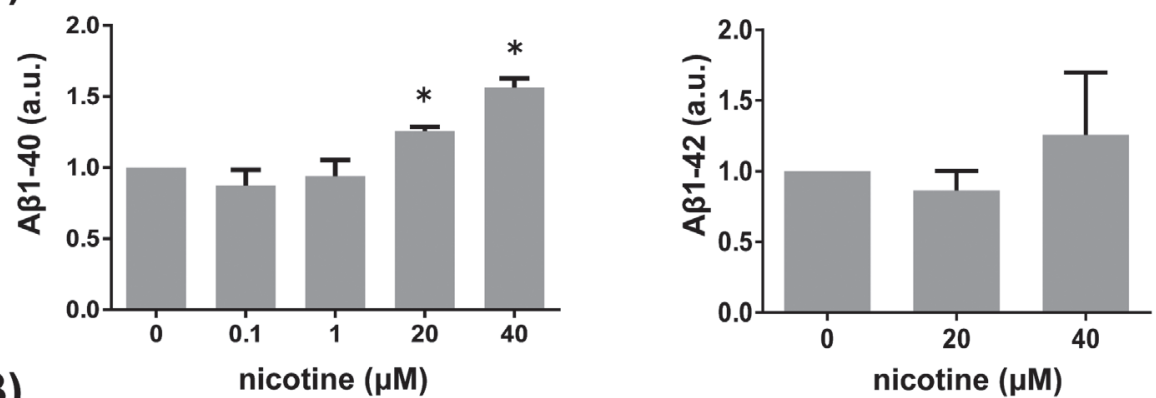

(B)
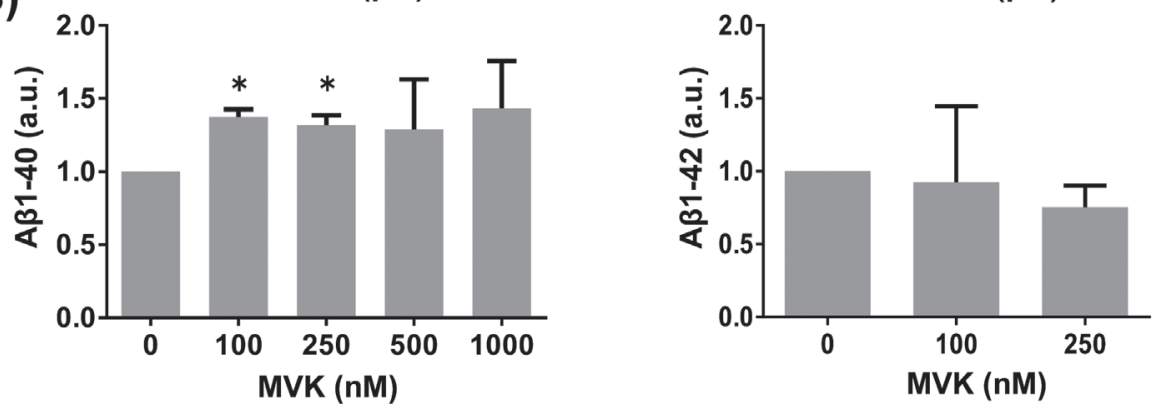

(C)

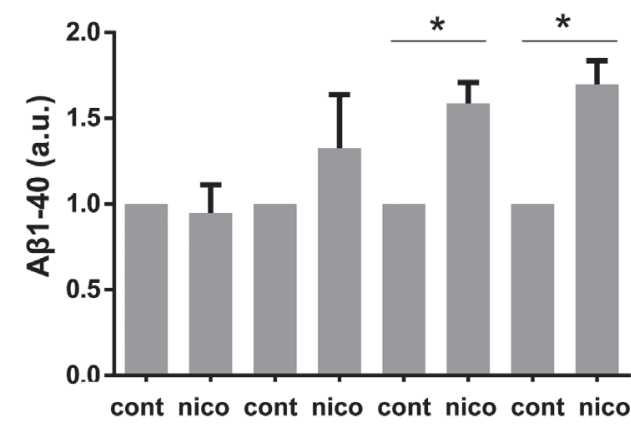

(D)
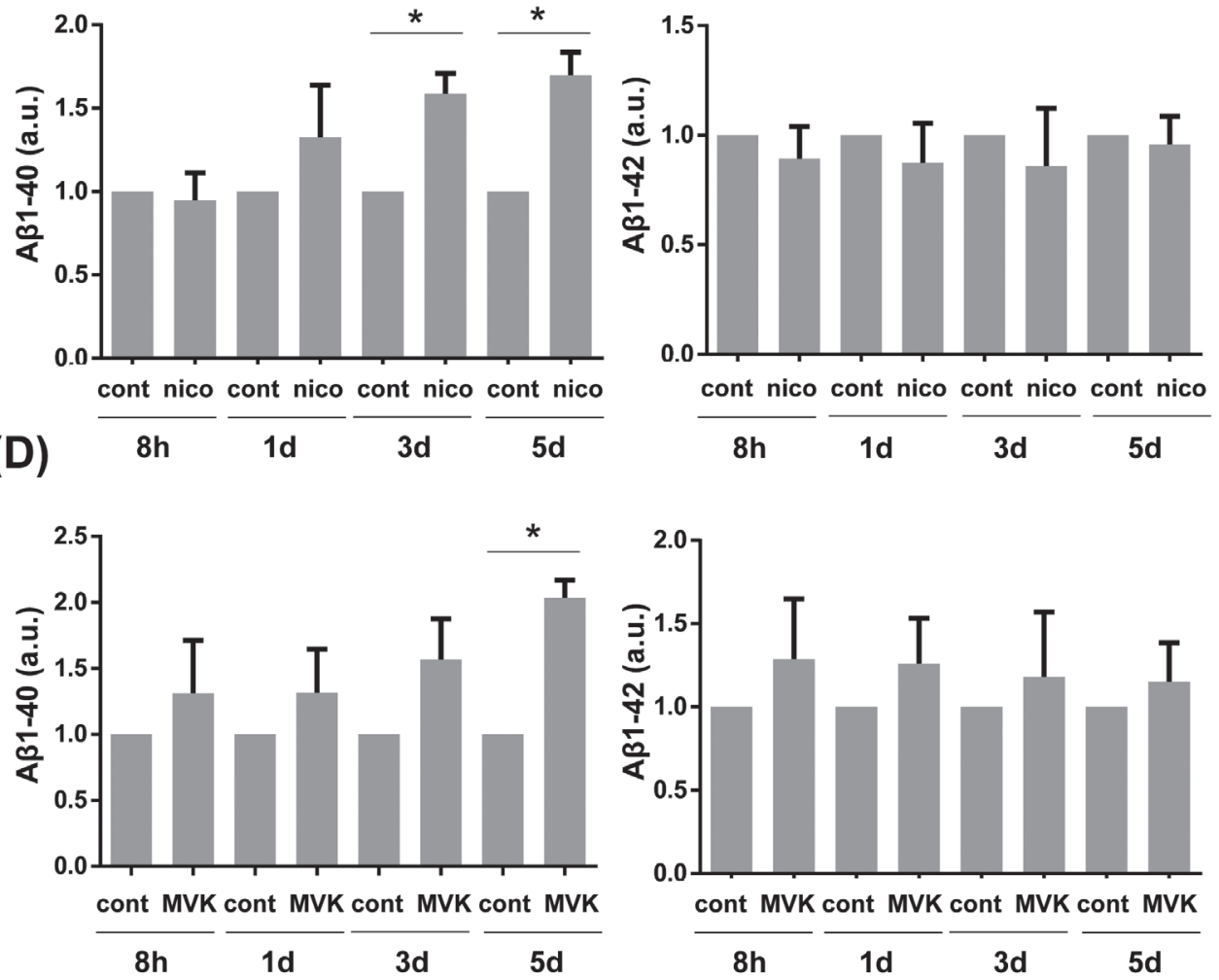

Fig. 2. Nicotine and MVK increased the extracellular level of A $\beta 1-40$ in wild-type APP-transfected cells. Levels of A $\beta 1-40$ and A $\beta 1-42$ in the culture media of cells incubated with nicotine or MVK were determined by ELISA. Cells were incubated with various concentrations of nicotine (A) or MVK (B) for 3 days. For C and D, cells were incubated with $20 \mu \mathrm{M}$ nicotine or $100 \mathrm{nM} \mathrm{MVK}$ for $8 \mathrm{hr}$ to 5 days. ${ }^{*} \mathrm{p}<0.05$, compared with the level in the culture media of control cells (cont). a.u.: arbitrary unit. 
(A)
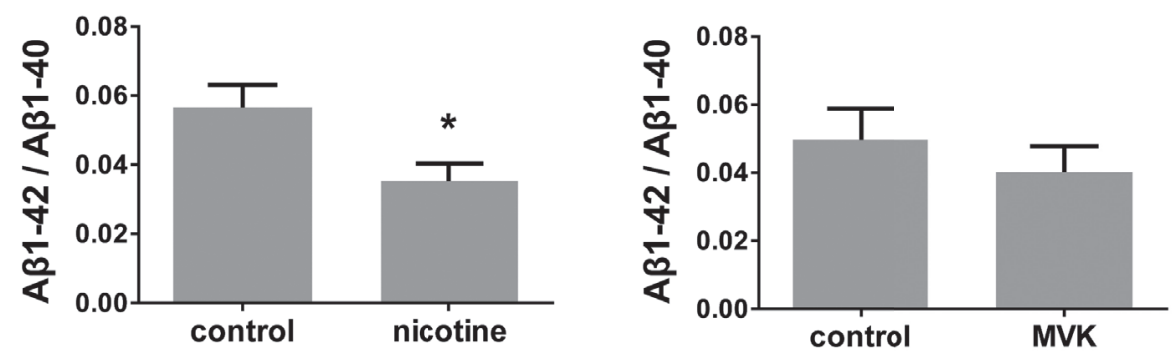

(B)
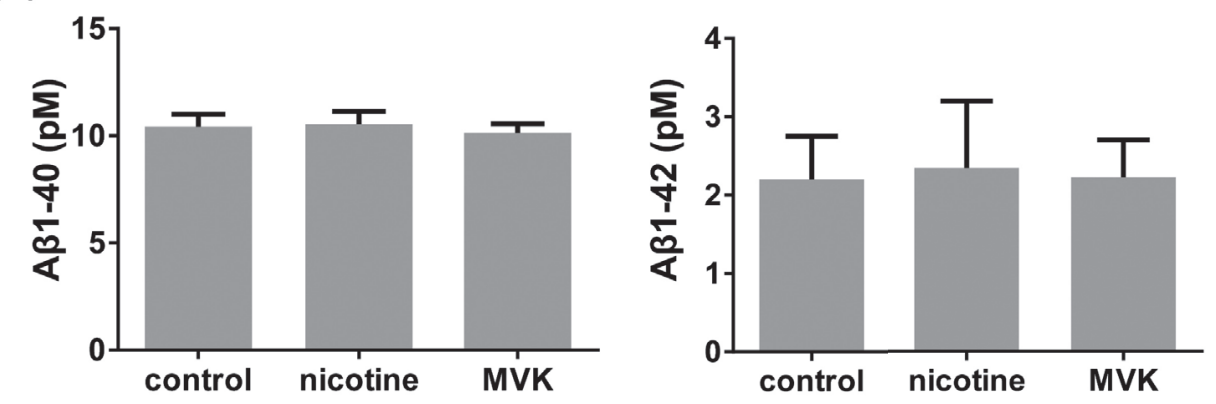

Fig. 3. The extracellular ratio of $A \beta 1-42$ to $A \beta 1-40$ and intracellular levels of $A \beta$ monomers in wild-type APP-transfected cells. (A) The ratio of A $\beta 1-42$ to A $\beta 1-40$ in the culture media of wild-type APP-transfected cells incubated with or without nicotine $(20 \mu \mathrm{M})$ or MVK $(100 \mathrm{nM})$ for 3 days. (B) The levels of A $\beta 1-40$ and A $\beta 1-42$ in cell lysates determined by ELISA. $* \mathrm{p}<0.05$, compared with the level in control cells.

SA system used in this study has been shown to detect high-molecular-weight A $\beta O$ s mainly composed of 45 - to 90-kDa oligomers (10-20 mers) (Fukumoto et al., 2010; Kasai et al., 2013, 2012). Accordingly, there is a possibility that the present detection system was not suited for the measurement of low-molecular-weight $\mathrm{A} \beta \mathrm{O}$ s which might be diminished due to the reduced ratio of extracellular $\mathrm{A} \beta 1-42 / \mathrm{A} \beta 1-40$ induced by nicotine.

Phosphorylation of APP at Thr 668 has been reported to regulate amyloidogenic processing of APP through facilitation of internalization and sorting of APP to early endocytic compartments where $\beta$-secretase processing occurs (Lee et al., 2003; Sodhi et al., 2008). In human neuroblastoma cells, phosphorylation of APP at Thr 668 was reported to promote $A \beta$ production independent of proteolytic processing by secretases (Araki et al., 2009). However, the level of phosphorylated APP was not altered in cells treated with nicotine, ruling out the possibility that nicotine affects phosphorylation of APP generated in transfected cells.

Nicotine significantly increased the levels of BACE1 in cells transfected with $A P P$. $\mathrm{A} \beta$ peptides are generated following the sequential cleavage of APP by BACE1 and $\gamma$-secretase, which is comprised of four subunits including presenilin, nicastrin, presenilin enhancer 2 , and anterior pharynx 1 (Hardy and Selkoe, 2002). The levels of A $\beta$ monomers in cell lysates were unchanged in cells treated with nicotine. This may be due to the rapid release of $A \beta$ monomers into the culture media once they are produced by the cleavage of APP on cellular membranes. Increased levels of BACE1 may facilitate cleavage of APP to expose the remaining C-terminal membrane-bound APP fragments that are subsequently cleaved by $\gamma$-secretase. Cleavage by $\gamma$-secretase occurs at several positions and inefficient cleavage generates longer A $\beta 1-42$ (Gibbons and Dean, 2016). The observed increase in extracellular $A \beta 1-40$ induced by nicotine might therefore be attributed to accelerated cleavage of APP by BACE. This is because accelerated cleavage of APP may promote ensuing $\gamma$-secretase cleavage to preferentially generate shorter $A \beta 1-40$. As for the significance of $\gamma$-secretase in the pathology of $\mathrm{AD}$, it was recently questioned due to the failure of the phase III clinical trial testing the inhibitor of $\gamma$-secretase (De Strooper, 2014; Doody et al., 2013). Cer- 
Cigarette smoke extracts increase protective amyloid- $\beta$ peptides

(A)

\author{
Swedish
}
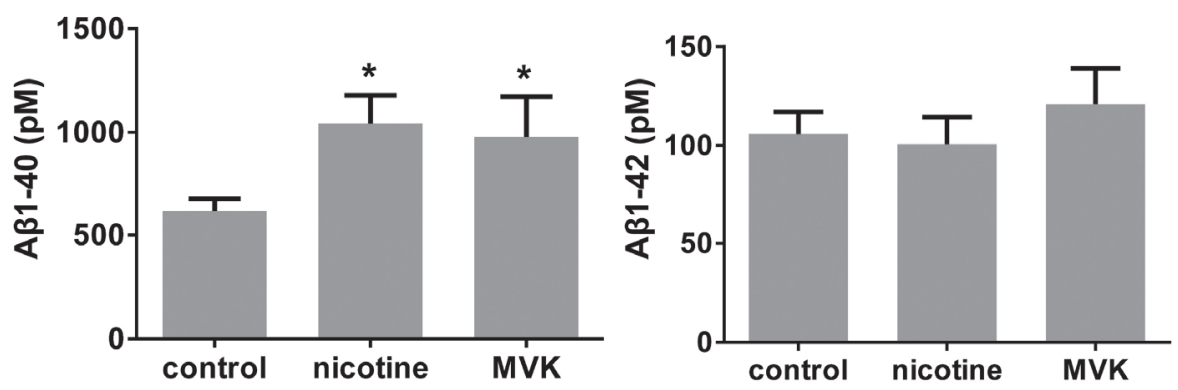

(B)
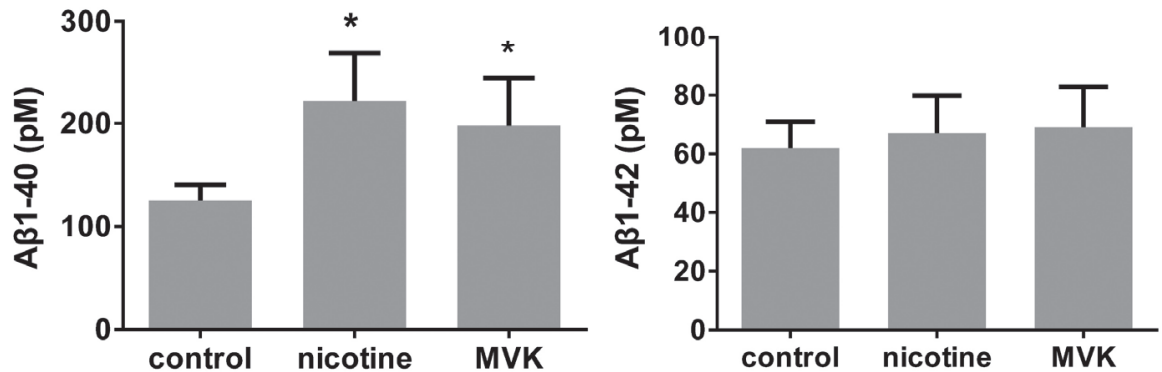

Fig. 4. Extracellular levels of $A \beta$ monomers in mutant APP-transfected cells. (A) Levels of A $\beta 1-40$ and $A \beta 1-42$ in the culture media of Swedish-type APP-transfected cells incubated with or without nicotine or MVK. (B) Levels of A $\beta 1-40$ and A $\beta 1-42$ in the culture media of London-type APP-transfected cells incubated with or without nicotine $(20 \mu \mathrm{M})$ or MVK $(100 \mathrm{nM})$ for 3 days. ${ }^{*} \mathrm{p}<0.05$, compared with the level in control cells.

(A)

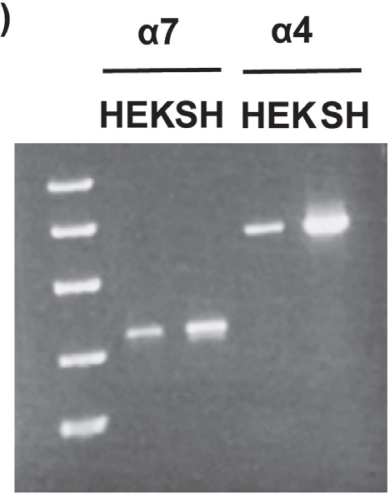

(B)

\title{
London
}


(A)

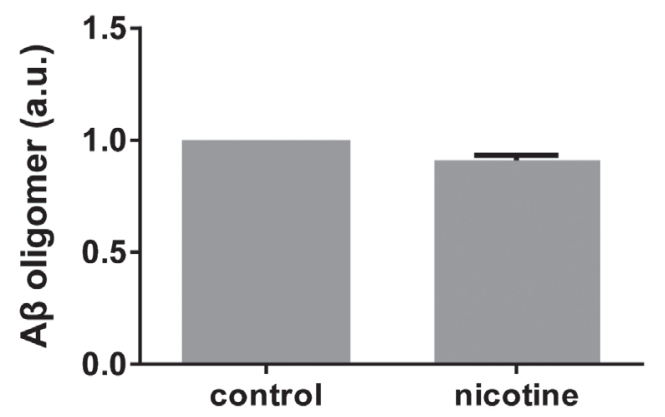

(C)

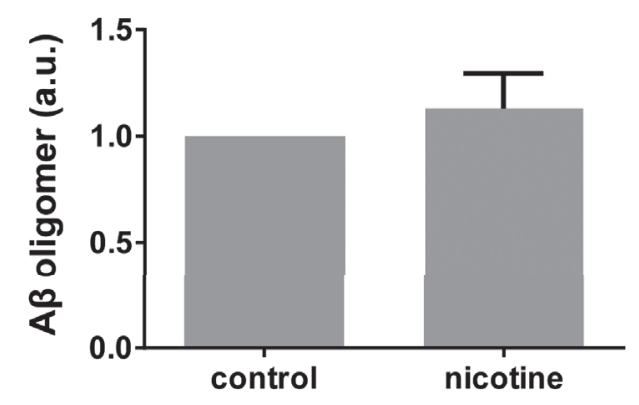

(B) Swedish

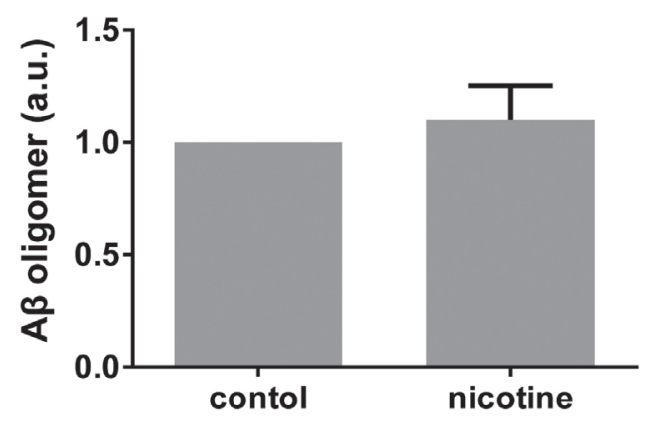

Fig. 6. Nicotine did not affect the levels of extracellular AßOs. (A) Levels of AßOs in the culture media of (A) wild-, (B) Swedishor (C) London-type APP-transfected cells were determined by A $\beta O$-specific ELISA. Cells were incubated with or without nicotine $(20 \mu \mathrm{M})$ or MVK $(100 \mathrm{nM})$ for 3 days. a.u.: arbitrary unit.

tainly, further investigation may be required on the relevance of $\gamma$-secretase in the generation of $\mathrm{A} \beta$ monomers.

Up to the present, protective effects of nicotine on AD development have been documented (Kihara et al., 1998). Several pathways involved in nicotine-mediated protection against $A \beta$ toxicity have been identified (Buckingham et al., 2009). Through the $\alpha 7-n A C h R / p h o s-$ phatidylinositol-3-kinase (PI3K) signaling pathway, nicotine demonstrated neuroprotective effects against $A \beta$ oligomer-induced damage in both pre- and postsynaptic regions (Inestrosa et al., 2013). In contrast with our findings, nicotine decreased BACE1 expression in a stable cell line expressing human APP and $\alpha 4 \beta 2-n A C h R$ (Nie et al., 2011). Another line of study demonstrated that chronic administration of nicotine reduced the levels of $A \beta 1$ 40 and BACE1 peptides in the hippocampal area CA1 of a rat model of AD (Srivareerat et al., 2011). The reason why these results differ from those we report here is cur- rently unknown. Because a regulated cellular system stably transfected with a single copy of $A P P$ was utilized in this study, the discrepancy could be attributed to the different culture or experimental system employed. However, our regulated cellular system that generates $A \beta$ species under relatively physiological conditions may provide fresh insight into the molecular mechanism underlying protective effects of nicotine against AD development.

In conclusion, a protective role against $\mathrm{AD}$ was demonstrated for nicotine and methyl vinyl ketone, major components of cigarette smoke extracts, by increasing the production of shorter $A \beta 1-40$ monomers to reduce the $A \beta 1-42 / A \beta 1-40$ ratio in a cellular model expressing human $A P P$. Our present findings may re-enforce the importance of the nAChR-mediated pathway in the neuroprotective action of nicotine, and aid in the development of disease-modifying therapies for this devastating neurodegenerative disorder. 
Cigarette smoke extracts increase protective amyloid- $\beta$ peptides
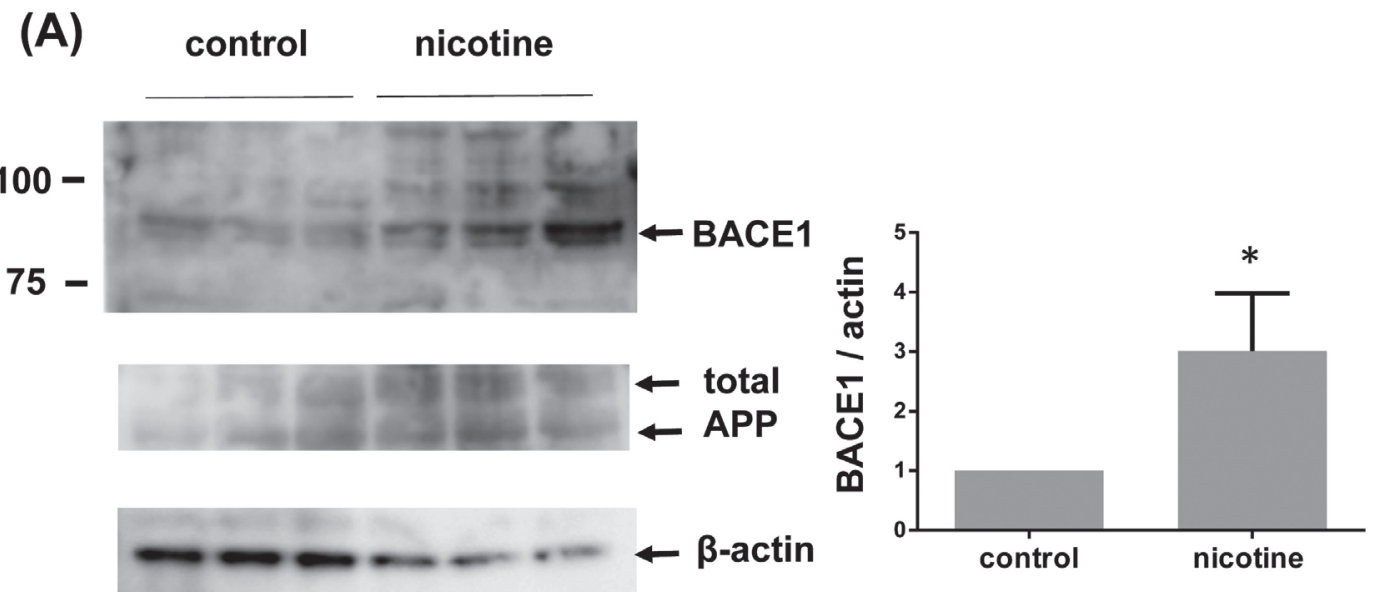

(B)
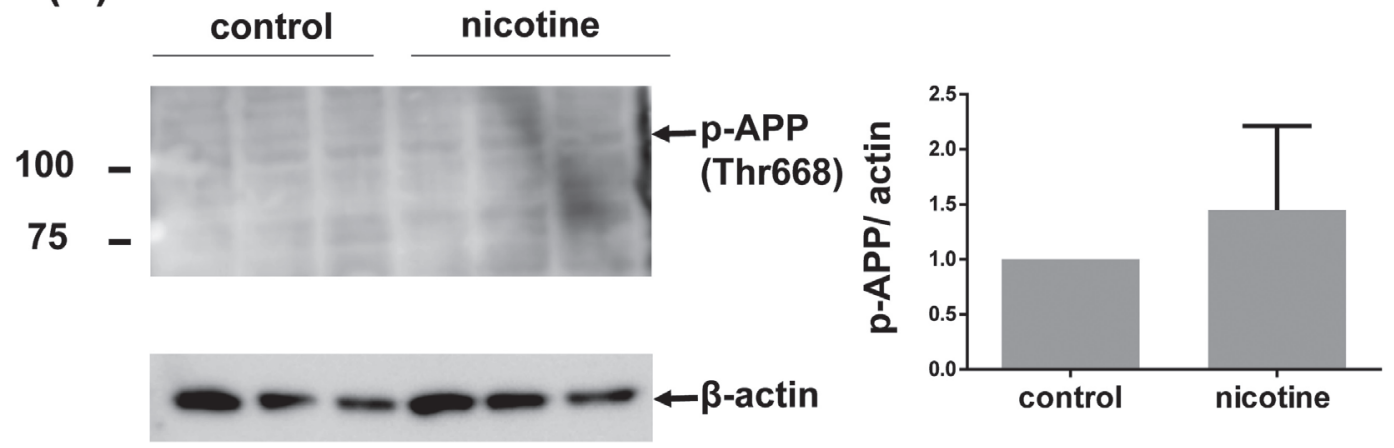

Fig. 7. Nicotine increased BACE1 that cleaves APP. Levels of BACE1 (A) or p-APP (B) in wild-type APP-transfected cells were determined by immunoblotting. The summed density of the bands was quantified. ${ }^{*} p<0.05$, compared with the level in control cells.

\section{ACKNOWLEDGMENTS}

This study was partly supported by a Grant-in-Aid for Scientific Research from the Ministry of Education, Culture, Sports, Science and Technology (25461288. To Y.O.) and by a grant from the Smoking Research Foundation of Japan (to C. Y-N.) .

Conflict of interest---- C. Y-N. is a recipient of the Smoking Research Foundation grant.

\section{REFERENCES}

Araki, W., Kume, H., Oda, A., Tamaoka, A. and Kametani, F. (2009): IGF-1 promotes beta-amyloid production by a secretaseindependent mechanism. Biochem. Biophys. Res. Commun., 380, 111-114.

Buckingham, S.D., Jones, A.K., Brown, L.A. and Sattelle, D.B. (2009): Nicotinic acetylcholine receptor signalling: roles in Alzheimer's disease and amyloid neuroprotection. Pharmacol.
Rev., 61, 39-61.

De Strooper, B. (2014): Lessons from a failed $\gamma$-secretase Alzheimer trial. Cell, 159, 721-726.

Doody, R.S., Raman, R., Farlow, M., Iwatsubo, T., Vellas, B., Joffe, S., Kieburtz, K., He, F., Sun, X., Thomas, R.G., Aisen, P.S., Siemers, E., Sethuraman, G. and Mohs, R.; Alzheimer's Disease Cooperative Study Steering Committee; Semagacestat Study Group. (2013): A phase 3 trial of semagacestat for treatment of Alzheimer's disease. N. Engl. J. Med., 369, 341-350.

Fukumoto, H., Tokuda, T., Kasai, T., Ishigami, N., Hidaka, H., Kondo, M., Allsop, D. and Nakagawa, M. (2010): High-molecular-weight beta-amyloid oligomers are elevated in cerebrospinal fluid of Alzheimer patients. FASEB J., 24, 2716-2726.

Gibbons, A. and Dean, B. (2016): The Cholinergic System: An Emerging Drug Target for Schizophrenia. Curr. Pharm. Des., 22, 2124-2133.

Hardy, J. and Selkoe, D.J. (2002): The amyloid hypothesis of Alzheimer's disease: progress and problems on the road to therapeutics. Science, 297, 353-356.

Hardy, J.A. and Higgins, G.A. (1992): Alzheimer's disease: the amyloid cascade hypothesis. Science, 256, 184-185.

Inestrosa, N.C., Godoy, J.A., Vargas, J.Y., Arrazola, M.S., Rios, J.A., Carvajal, F.J., Serrano, F.G. and Farias, G.G. (2013): Nic- 
Y. Ohshima et al.

otine prevents synaptic impairment induced by amyloid- $\beta$ oligomers through $\alpha 7$-nicotinic acetylcholine receptor activation. Neuromolecular Med., 15, 549-569.

Kasai, T., Tokuda, T., Taylor, M., Kondo, M., Mann, D.M., Foulds, P.G., Nakagawa, M. and Allsop, D. (2013): Correlation of A $\beta$ oligomer levels in matched cerebrospinal fluid and serum samples. Neurosci. Lett., 551, 17-22.

Kasai, T., Tokuda, T., Taylor, M., Nakagawa, M. and Allsop, D. (2012): Utilization of a multiple antigenic peptide as a calibration standard in the BAN50 single antibody sandwich ELISA for $A \beta$ oligomers. Biochem. Biophys. Res. Commun., 422, 375 380.

Kihara, T., Shimohama, S., Urushitani, M., Sawada, H., Kimura, J., Kume, T., Maeda, T. and Akaike, A. (1998): Stimulation of alpha4beta2 nicotinic acetylcholine receptors inhibits beta-amyloid toxicity. Brain Res., 792, 331-334.

Lee, M.S., Kao, S.C., Lemere, C.A., Xia, W., Tseng, H.C., Zhou, Y., Neve, R., Ahlijanian, M.K. and Tsai, L.H. (2003): APP processing is regulated by cytoplasmic phosphorylation. J. Cell Biol., 163, 83-95.

Lombardo, S. and Maskos, U. (2015): Role of the nicotinic acetylcholine receptor in Alzheimer's disease pathology and treatment. Neuropharmacology, 96 (Pt B), 255-262.

Muresan, V. and Ladescu Muresan, Z. (2015): Amyloid- $\beta$ precursor protein: multiple fragments, numerous transport routes and mechanisms. Exp. Cell Res., 334, 45-53.

Nie, H.Z., Li, Z.Q., Yan, Q.X., Wang, Z.J., Zhao, W.J., Guo, L.C. and Yin, M. (2011): Nicotine decreases beta-amyloid through regulating BACE1 transcription in SH-EP1- $\alpha 4 \beta 2 \mathrm{nAChR}-$ APP695 cells. Neurochem. Res., 36, 904-912.

Nilsson, P., Saito, T. and Saido, T.C. (2014): New Mouse Model of Alzheimer's. ACS Chem. Neurosci., 5, 499-502.

Ono, K., Hasegawa, K., Yamada, M. and Naiki, H. (2002): nicotine breaks down preformed Alzheimer's beta-amyloid fibrils in vitro.
Biol. Psychiatry, 52, 880-886.

Saido, T.C. (2013): Metabolism of amyloid $\beta$ peptide and pathogenesis of Alzheimer's disease. Proc. Jpn. Acad., Ser. B, Phys. Biol. Sci., 89, 321-339.

Saito, T., Matsuba, Y., Mihira, N., Takano, J., Nilsson, P., Itohara, S., Iwata, N. and Saido, T.C. (2014): Single App knock-in mouse models of Alzheimer's disease. Nat. Neurosci., 17, 661-663.

Scheuner, D., Eckman, C., Jensen, M., Song, X., Citron, M., Suzuki, N., Bird, T.D., Hardy, J., Hutton, M., Kukull, W., Larson, E., Levy-Lahad, E., Viitanen, M., Peskind, E., Poorkaj, P., Schellenberg, G., Tanzi, R., Wasco, W., Lannfelt, L., Selkoe, D. and Younkin, S. (1996): Secreted amyloid beta-protein similar to that in the senile plaques of Alzheimer's disease is increased in vivo by the presenilin 1 and 2 and APP mutations linked to familial Alzheimer's disease. Nat. Med., 2, 864-870.

Selkoe, D.J. (1991): The molecular pathology of Alzheimer's disease. Neuron, 6, 487-498.

Selkoe, D.J. (1997): Alzheimer's disease: genotypes, phenotypes, and treatments. Science, 275, 630-631.

Shaw, S., Bencherif, M. and Marrero, M.B. (2002): Janus kinase 2, an early target of alpha 7 nicotinic acetylcholine receptor-mediated neuroprotection against Abeta-(1-42) amyloid. J. Biol. Chem., 277, 44920-44924.

Sodhi, C.P., Perez, R.G. and Gottardi-Littell, N.R. (2008): Phosphorylation of beta-amyloid precursor protein (APP) cytoplasmic tail facilitates amyloidogenic processing during apoptosis. Brain Res., 1198, 204-212.

Srivareerat, M., Tran, T.T., Salim, S., Aleisa, A.M. and Alkadhi, K.A. (2011): Chronic nicotine restores normal $A \beta$ levels and prevents short-term memory and E-LTP impairment in $A \beta$ rat model of Alzheimer's disease. Neurobiol. Aging, 32, 834-844.

Watanabe-Hosomi, A., Watanabe, Y., Tanaka, M., Nakagawa, M. and Mizuno, T. (2012): Transendocytosis is impaired in CADASIL-mutant NOTCH3. Exp. Neurol., 233, 303-311. 